\title{
Assessing the role of the TREM2 p.R47H variant as a risk factor for Alzheimer's disease and frontotemporal dementia
}

Agustín Ruiz ${ }^{\mathrm{a}}$, Oriol Dols-Icardo ${ }^{\mathrm{b}, \mathrm{c}}, \mathrm{M}^{\mathrm{a}}$ Jesús Bullido $^{\mathrm{c}, \mathrm{d}, \mathrm{e}}$, Pau Pastor ${ }^{\mathrm{c}, \mathrm{f,g}}$, Eloy Rodríguez-Rodríguez $^{\mathrm{c}, \mathrm{h}}$, Adolfo López de Munain ${ }^{\mathrm{c}, \mathrm{i}}$, Marian M. de Pancorbo ${ }^{\mathrm{j}}$, Jordi Pérez-Tur ${ }^{\mathrm{c}, \mathrm{k}}$, Victoria Álvarez, Anna Antonell ${ }^{\mathrm{m}}$, Jesús López-Arrieta ${ }^{\mathrm{e}, \mathrm{n}}$, Isabel Hernández $^{\mathrm{a}}$, Lluís Tárraga ${ }^{\mathrm{a}}$, Mercè Boada ${ }^{\mathrm{a}, \mathrm{o}}$, Alberto Lleóo ${ }^{\mathrm{b}, \mathrm{c}}$, Rafael Blesa ${ }^{\mathrm{b}, \mathrm{c}}$, Ana

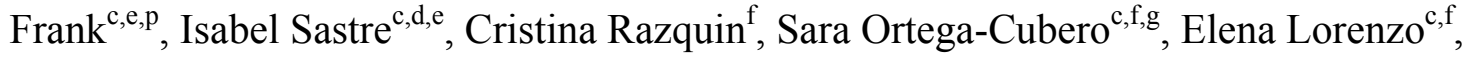

Pascual Sánchez-Juan ${ }^{\text {c,h }}$, Onofre Combarros ${ }^{\text {c,h }}$, Fermín Moreno ${ }^{\text {c,i }}$, Ana Gorostidi ${ }^{\mathrm{c}, \mathrm{i}}$, Miquel Baquero $^{\mathrm{q}}$, Eliecer Coto ${ }^{1}$, Raquel Sánchez-Valle ${ }^{\mathrm{m}}$, Jordi Clarimón ${ }^{\mathrm{b}, \mathrm{c},{ }^{*}}$, on behalf of The dementia genetic Spanish consortium (DEGESCO)

${ }^{a}$ Alzheimer Research Center and Memory Clinic. Fundació ACE. Institut Català de Neurociències Aplicades. Barcelona, Spain.

${ }^{\mathrm{b}}$ Neurology Department, IIB-Sant Pau, Hospital de la Santa Creu i Sant Pau, Barcelona, Spain.

${ }^{\mathrm{c}}$ CIBERNED, Center for Networked Biomedical Research into Neurodegenerative Diseases, Madrid, Spain.

${ }^{\mathrm{d}}$ Centro de Biología Molecular Severo Ochoa (CSIC-UAM), Madrid, Spain.

e Institute of Sanitary Research “'Hospital la Paz”' (IdIPaz), Madrid, Spain.

${ }^{\mathrm{f}}$ Neurogenetics Laboratory, Division of Neurosciences, Center for Applied Medical Research, University of Navarra, Pamplona, Spain.

g Department of Neurology, Clínica Universidad de Navarra, University of Navarra, School of Medicine, Pamplona, Spain. 
${ }^{\mathrm{h}}$ Neurology Service, University Hospital Marqués de Valdecilla (University of Cantabria and IFIMAV), Santander, Spain.

${ }^{i}$ Neuroscience Area. Institute Biodonostia, and Department of Neurosciences, University of Basque Contry EHU-UPV San Sebastián, Spain.

${ }^{j}$ Centro de Investigación "Lascaray" Ikergunea, Universidad del País Vasco, VitoriaGasteiz, Spain.

${ }^{\mathrm{k}}$ Institut de Biomedicina de Valencia-CSIC. Valencia, Spain.

${ }^{1}$ Molecular Genetics Laboratory, Genetics Unit, Hospital Universitario Central de Asturias, Oviedo, Spain.

m Alzheimer's disease and other cognitive disorders, Neurology Department, Hospital Clínic, IDIBAPS, Barcelona, Spain.

${ }^{\mathrm{n}}$ Memory Unit, University Hospital La Paz-Cantoblanco, Madrid, Spain.

${ }^{0}$ Hospital Universitari Vall d'Hebron - Institut de Recerca, Universitat Autònoma de Barcelona (VHIR-UAB), Barcelona, Spain.

${ }^{\mathrm{p}}$ Neurology Service, Hospital Universitario La Paz (UAM), Madrid, Spain.

${ }^{\mathrm{q}}$ Neurology Service, Hospital Universitari i Politècnic La Fe, València, Spain

\section{Corresponding Author:}

Jordi Clarimón, Genetics of Neurodegenerative Disorders Unit, IIB-Sant Pau, Neurology Department, Hospital de la Santa Creu i Sant Pau. Sant Antoni M. Claret 167, 08025 Barcelona, Spain email: jclarimon@santpau.cat; Fax num. +34935565602 ; Tel num. +34932919050 ext.8230 


\section{ABSTRACT}

A non-synonymous genetic rare variant, rs75932628-T (p.R47H), in the TREM2 gene has recently been reported to be a strong genetic risk factor for Alzheimer's disease (AD). Also, rare recessive mutations have been associated with frontotemporal dementia (FTD). We aimed to investigate the role of $\mathrm{p} . \mathrm{R} 47 \mathrm{H}$ variant in AD and FTD through a multi-center study comprising 3,172 AD and 682 FTD patients and 2,169 healthy controls from Spain. We found that $0.6 \%$ of $\mathrm{AD}$ cases carried this variant compared to $0.1 \%$ of controls (odds ratio $[\mathrm{OR}]=4.12,95 \%$ confidence interval $[\mathrm{CI}]$ : 1.21-14.00, $P=0.014)$. A meta-analysis comprising 32,598 subjects from four previous studies demonstrated the large effect of the p.R $47 \mathrm{H}$ variant in $\mathrm{AD}$ risk $(\mathrm{OR}=4.11,95 \%$ CI: $\left.2.99-5.68, P=5.27 \times 10^{-18}\right)$. We did not find an association between $\mathrm{p} . \mathrm{R} 47 \mathrm{H}$ and age of onset of AD or family history of dementia. Finally, none of the FTD patients harbored this genetic variant. These data strongly support the important role of p.R47H in $\mathrm{AD}$ risk and suggest that this rare genetic variant is not related to FTD.

Key Words: Alzheimer's disease; Frontotemporal dementia; TREM2; Genetic association; p.R47H; Rare variant 


\section{Introduction}

The rapid population growth in recent centuries and the evolutionary forces that shape allelic variation over time have led to a large number of rare genetic variants in the human genome, and they seem to vastly exceed the number of common alleles (Tennessen et al. 2012). A substantial fraction of these rare variants may have functional consequences and therefore can contribute to the allelic architecture of complex diseases (Pritchard, 2001). A very recent example of a rare genetic variant with large individual effect is the non-synonymous amino acid substitution p. $\mathrm{R} 47 \mathrm{H}$ (rs75932628) in the gene encoding the triggering receptor expressed on myeloid cells 2 (TREM2) and its association with the risk for sporadic Alzheimer's disease (AD) (Guerreiro et al. 2013; Jonsson et al. 2013). This association has also been replicated in a cohort of French patients with an early-onset form of AD (EOAD, age of onset $\leq 65$ years) (Pottier et al. 2013), and in a Spanish study with a series of patients that comprised both late and EOAD (Benitez et al. 2013). Interestingly, homozygous mutations in TREM2 have been described in patients with an unusual early-onset frontotemporal dementia (FTD) from consanguineous families living in the Anatolian region of Turkey (Guerreiro et al. 2013) and in a Colombian family (Giraldo et al. 2013), thus broadening the clinical heterogeneity spectrum caused by mutations in this particular locus. In addition, homozygous mutations in TREM2 are also the cause of Nasu-Hakola disease, which is mainly characterized by pre-senile FTD and cystic bone lesions (Paloneva et al. 2002).

Since (i) the TREM2 p.R47H variant has been related to the broad spectrum of AD, including EOAD, (ii) no specific assessment of this variant has been performed in FTD, and (iii) allele frequency of the p.R47H varies across populations, we aimed to evaluate how this non-synonymous amino acid change contributes to the risk of early- 
and late-onset forms of AD or FTD by studying a large cohort of patients and controls from Spanish origin.

\section{Methods}

\subsection{Study subjects}

A total of 3,172 AD patients (mean age at onset $74.8 \pm 9.9$ years, $68.5 \%$ women), 682 FTD patients (mean age at onset $64.8 \pm 9.9$ years, $46.8 \%$ women), and 2,169 healthy controls (mean age at clinical assessment $75.4 \pm 10.3$ years, $58.2 \%$ women) were collected through a collaborative effort involving 11 specialized centers across the country. All individuals were Spanish and of European origin. Among AD patients, 466 were classified as early-onset cases (EOAD) (cut-off age of onset being 65 years). Patients were diagnosed using established clinical criteria for AD (McKhann et al. 1984) or FTD (Neary et al. 1998). All participants or their families provided written informed consent, and the study was approved by the respective ethics committees.

\subsection{Genotyping}

Genotyping of the rs75932628 (p.R47H) variant was performed using three approaches: TaqMan SNP Genotyping Assays (Applied Biosystems, Foster city, California), High Resolution Melting (Eco-PCR, Illumina, San Diego, California), and KASPar (KBioscience, Berlin). A human DNA sample carrying a T-allele (rs75932628T) in heterozygous state was distributed to all genotyping centers and was included as a positive control in all genotyping plates. 


\subsection{Statistical analysis}

Genotype frequency comparisons were performed by Fisher's exact test.

Differences between groups were analyzed by Student's t-test for continuous data. Multiple logistic regression models were used to adjust for covariates, such as age, gender and $A P O E-\varepsilon 4$ status. Data were analyzed using the Statistical Package for the Social Sciences, version 19.0.0 (SPSS Inc., Chicago, IL). Genetic interactions (epistasis) were assessed by the synergy factor analysis (Cortina-Borja et al. 2009). Meta-analysis was conducted using the inverse variance method (fixed effects model) in Episheet excel application according to Fleiss (Fleiss 1993). Fixed and random effects meta-analyses were automatically generated by Episheet. The weighting of each study was calculated using the estimated standard errors. The bona fide genome-wide significant $P$-value threshold was established at $P<5 \times 10^{-8}$ (de Bakker et al. 2008). Final meta-analysis results and Forest plot for TREM2 p.R47H showing association results in all available data were derived using OpenMeta (Wallace et al. 2009). Power calculations were performed with the PS software (Version 2.1.30).

\section{Results}

A total of 6,023 individuals were genotyped for the $\mathrm{p} . \mathrm{R} 47 \mathrm{H}$ polymorphism, and distribution of genotype frequencies across phenotypes is presented in Table 1 . The T allele at the rs 75932628 biallelic polymorphism was carried by $0.6 \%$ of AD cases and $0.1 \%$ of controls $(P=0.014)$, and yielded an odds ratio $(\mathrm{OR})$ of 4.12 [95\% confidence interval [CI]: 1.21-14.00], almost identical to the risk conferred by the $A P O E$ - $\varepsilon 4$ allele (4.2, Table 1). Although not statistically significant, age at onset of AD was slightly lower in rs75932628- $\mathrm{T}$ carriers than noncarriers $(70 \pm 6.0$ and $74.8 \pm 9.9$ years, respectively, $P=0.08$ ). To effectively detect whether the association between 
rs75932628-T variant and AD was modified by age, the sample was stratified into EOAD cases (age of onset $\leq 65$ years old, $n=466$ ) and late-onset cases ( $>65$ years old, $\mathrm{n}=2,375)$. The rs75932628-T association was replicated within the late-onset AD series $(\mathrm{OR}=4.28 ; 95 \% \mathrm{CI}: 1.23-14.92, P=0.014)$. However, no significant association was found for the EOAD series $(\mathrm{OR}=3.12 ; 95 \% \mathrm{CI}$ : 0.52-18.68, $P=0.217)$. Data regarding family history of dementia were available for 14/18 AD patients carrying the rs75932628-T allele. Of these patients, only five (35.7\%) reported a positive family history of dementia. This percentage was almost identical to the prevalence of family history of dementia in rs75932628-T noncarriers $(35.3 \%, P=0.974)$. To further address the association between the p.R47H variant and the risk of $\mathrm{AD}$, and to compare the observed effect size of $\mathrm{p} . \mathrm{R} 47 \mathrm{H}$ among different populations, we conducted a metaanalysis using our data and the available data from the literature (Benitez et al. 2013; Guerreiro et al. 2013; Jonsson et al. 2013; Pottier et al. 2013). In total, we analyzed the genotypes of 32,598 subjects comprising 12,967 patients and 19,631 controls. The summary OR under a fixed effect model showed that individuals with the $\mathrm{p} . \mathrm{R} 47 \mathrm{H}$ variant were 4.11 times more likely to develop AD than noncarriers (95\% CI: 2.99-5.68, $P=5.27 \times 10^{-18}$, Figure 1$)$. No evidence of heterogeneity between studies was detected $(\mathrm{Q}=2.17, P>0.98$ with nine degrees of freedom $)$.

We next assessed the effect of the $A P O E$ - $\varepsilon 4$ allele on the association between rs75932628-T and the risk of AD. The risk of the $\mathrm{p} . \mathrm{R} 47 \mathrm{H}$ variant was no longer significant after adjustment for the $A P O E-\varepsilon 4$ allele $(P=0.34)$. Since there were significant differences between the frequency of rs75932628-T in carriers of the APOE$\varepsilon 4$ allele compared to noncarriers $(P=0.007)$, we then evaluated whether the TREM2 association was indeed confounded by the presence of $A P O E-\varepsilon 4$ in rs $75932628-T$ carriers through a meta-analysis that included two previous studies with six populations 
(Jonsson et al. 2013; Pottier et al. 2013). This analysis showed no difference in the frequency of rs 75932628 - $\mathrm{T}$ carriers according to $A P O E-\varepsilon 4$ status $(P=0.28$, Suppl. Fig. 1). No significant interaction between $A P O E-\varepsilon 4$ and rs $75932628-\mathrm{T}$ resulted from the logistic-regression model $(P=0.577)$. Evaluation of this interaction by the synergy factor $(S F)$ analysis, which measures both the size and significance of genetic interactions (Cortina-Borja et al. 2009), corroborated the lack of synergy between $A P O E-\varepsilon 4$ and rs75932628-T $(S F=1.31, P=0.43)$.

The study of the p.R47H variant in the FTD group $(n=682)$ did not disclose any carrier of this genetic variant.

\section{Discussion}

The role of rare genetic variants in common disorders has been fueled in the last few years by the appearance of next-generation sequencing technologies. Two independent works have recently demonstrated the power of these techniques. After a large scale analysis of sequencing data, they identified the rare $\mathrm{p} . \mathrm{R} 47 \mathrm{H}$ substitution as a major genetic risk factor for AD (Guerreiro et al. 2013; Jonsson et al. 2013). In the present work we were able to replicate this finding by analyzing a large case-control cohort comprising more than 6,000 individuals. The effect size of the TREM2 rs75932628-T rare variant was consistent with those reported previously (Guerreiro et al. 2013; Jonsson et al. 2013; Benitez et al. 2013), and reached the same magnitude as the $A P O E-\varepsilon 4$ allele, the most important genetic risk factor for AD. Our meta-analysis comprising $>32,000$ subjects strongly supports the important role of the $\mathrm{p} . \mathrm{R} 47 \mathrm{H}$ nonsynonymous variant in $\mathrm{AD}$ risk.

We were not able to replicate the recently reported association of rs $75932628-\mathrm{T}$ and EOAD risk in a French population (Pottier et al. 2013), despite the frequency of 
EOAD patients carrying the p.R47H variant being slightly higher than controls $(0.4 \%$ and $0.1 \%$, respectively). A possible explanation for this lack of replication is that the frequency of the rs75932628-T variant in the Spanish population is much lower than that of the French population $(0.14 \%$ and $0.5 \%$, respectively in controls, $0.4 \%$ and $2.0 \%$ in patients). Therefore, even though our sample size was almost double that of Pottier et al., the much lower frequency of the rs75932628-T in our population could have led to an insufficient statistical power. The present study had a power of $46 \%$ (alpha $=0.05$ ) to detect a risk equal to that reported in the French analysis. It is important to note, however, that patients carrying the rs75932628-T variant had a slightly, although not significantly, earlier age of onset, thus raising the question as to whether this genetic variant could modulate the age of $\mathrm{AD}$ onset.

We did not find any interaction between the $\varepsilon 4$ allele of the $A P O E$ gene and the p.R47H variant. Nevertheless, the $A P O E$ - $\varepsilon 4$ adjusted risk of the rs 75932628 -T variant resulted in a loss of statistical significance. The high prevalence of the APOE-E4 isoform in $\mathrm{AD}$ patients, in conjunction with its prominent effect on $\mathrm{AD}$ risk may explain this somewhat unexpected outcome. However, our meta-analysis to evaluate the effect of $A P O E-\varepsilon 4$ on the association of rs75932628-T with AD strongly indicates the independence of both genes in the risk of AD. Therefore, co-occurrence of both alleles (rs75932628-T and APOE- $\varepsilon 4$ ) in our AD series was not confirmed in other populations and might be due to random effects.

As far as we know, this is the first study to assess the role of the p. $\mathrm{R} 47 \mathrm{H}$ variant in FTD patients. Our results strongly suggest that being a heterozygous carrier of this variant does not contribute to the risk of common forms of FTD and emphasize the clinical heterogeneity associated with TREM2. In-depth analysis of this locus in FTD is warranted. 


\section{Disclosure statement}

The authors declare no potential conflicts of interest.

\section{Acknowledgments}

We would like to thank patients, their families, and controls for their participation in the study. This study was supported by grants from Instituto de Salud Carlos III (PI12/01311 and 12/00013), grants from the Ministry of Science (SAF2010-15558, SAF2009-10434), Centro de Investigación Biomédica en Red sobre Enfermedades Neurodegenerativas (CIBERNED, Spain), Consolider (CSD2010-00045), and the Department of Health of the Government of Navarra (refs. 13085 and 3/2008). CR held during the period 2009-2013 a "Torres Quevedo" fellowship from the Spanish Ministry of Science and Technology, co-financed by the European Social Fund. Fundació ACE researchers are indebted to Trinitat Port-Carbó and her family who are supporting Fundació ACE scientific programs. We also would like to thank Drs. P. Gil and P. Coria for their cooperation in the generation of the case-control samples, and Drs. José Luis Molinuevo, Albert Lladó, and Lorena Rami (from Hospital Clínic), Drs. Juan Fortea and Daniel Alcolea (from Hospital Sant Pau), Dr. J.A. Burguera (from Hospital Universitari i Politècnic La Fe) and Dr. Antonio Salazar (from Direcció General de Salut Pública de la Generalitat Valenciana) for their effort in clinical evaluation and sample recruitment, and Carolyn Newey for Editorial help. 


\section{References}

Benitez, B.A., Cooper, B., Pastor, P., Jin, S.C., Lorenzo, E., Cervantes, S., Cruchaga, C., 2013. TREM2 is associated with the risk of Alzheimer's disease in Spanish population. Neurobiol. Aging 34, 1711 e15-17.

Cortina-Borja, M., Smith, A.D., Combarros, O., Lehmann, D.J., 2009. The synergy factor: a statistic to measure interactions in complex diseases. BMC Res. Notes 2, 105.

de Bakker, P.I., Ferreira, M.A., Jia, X., Neale, B.M., Raychaudhuri, S., Voight, B.F., 2008. Practical aspects of imputation-driven meta-analysis of genome-wide association studies. Hum. Mol. Genet. 17, R122-128.

Fleiss, J.L., 1993. The statistical basis of meta-analysis. Stat. Methods Med. Res. 2, 121-145.

Giraldo, M., Lopera, F., Siniard, A.L., Corneveaux, J.J., Schrauwen, I., Carvajal, J., Muñoz, C., Ramírez-Restrepo, M., Gaiteri, C., Myers, A.J., Caselli, R.J., Kosik, K.S., Reiman, E.M., Huentelman, M.J., 2013. Variants in triggering receptor expressed on myeloid cells 2 are associated with both behavioral variant frontotemporal lobar degeneration and Alzheimer's disease. doi: 10.1016/j.neurobiolaging.2013.02.016.

Guerreiro, R., Wojtas, A., Bras, J., Carrasquillo, M., Rogaeva, E., Majounie, E., Cruchaga, C., Sassi, C., Kauwe, J.S., Younkin, S., Hazrati, L., Collinge, J., Pocock, J., Lashley, T., Williams, J., Lambert, J.C., Amouyel, P., Goate, A., Rademakers, R., Morgan, K., Powell, J., St George-Hyslop, P., Singleton, A., Hardy, J., 2013.

TREM2 variants in Alzheimer's disease. N. Engl. J. Med. 368, 117-127.

Guerreiro, R.J., Lohmann, E., Bras, J.M., Gibbs, J.R., Rohrer, J.D., Gurunlian, N., Dursun, B., Bilgic, B., Hanagasi, H., Gurvit, H., Emre, M., Singleton, A., Hardy, J., 
2013. Using exome sequencing to reveal mutations in TREM2 presenting as a frontotemporal dementia-like syndrome without bone involvement. JAMA Neurol. $70,78-84$.

Jonsson, T., Stefansson, H., Steinberg, S., Jonsdottir, I., Jonsson, P.V., Snaedal, J., Bjornsson, S., Huttenlocher, J., Levey, A.I., Lah, J.J., Rujescu, D., Hampel, H., Giegling, I., Andreassen, O.A., Engedal, K., Ulstein, I., Djurovic, S., IbrahimVerbaas, C., Hofman, A., Ikram, M.A., van Duijn, C.M., Thorsteinsdottir, U., Kong, A., Stefansson, K., 2013. Variant of TREM2 associated with the risk of Alzheimer's disease. N. Engl. J. Med. 368, 107-116.

McKhann, G., Drachman, D., Folstein, M., Katzman, R., Price, D., Stadlan, E.M., 1984. Clinical diagnosis of Alzheimer's disease: report of the NINCDS-ADRDA Work Group under the auspices of Department of Health and Human Services Task Force on Alzheimer's Disease. Neurology 34, 939-944.

Neary, D., Snowden, J.S., Gustafson, L., Passant, U., Stuss, D., Black, S., Freedman, M., Kertesz, A., Robert, P.H., Albert, M., Boone, K., Miller, B.L., Cummings, J., Benson, D.F., 1998. Frontotemporal lobar degeneration: a consensus on clinical diagnostic criteria. Neurology 51, 1546-1554.

Paloneva, J., Manninen, T., Christman, G., Hovanes, K., Mandelin, J., Adolfsson, R., Bianchin, M., Bird, T., Miranda, R., Salmaggi, A., Tranebjaerg, L., Konttinen, Y., Peltonen, L., 2002. Mutations in two genes encoding different subunits of a receptor signaling complex result in an identical disease phenotype. Am. J. Hum. Genet. 71, $656-662$.

Pottier, C., Wallon, D., Rousseau, S., Rovelet-Lecrux, A., Richard, A.C., Rollin-Sillaire, A., Frebourg, T., Campion, D., Hannequin, D., 2013. TREM2 R47H Variant as a Risk Factor for Early-Onset Alzheimer's Disease. J. Alzheimers Dis. 35, 45-49. 
Pritchard, J.K., 2001. Are rare variants responsible for susceptibility to complex diseases? Am. J. Hum. Genet. 69, 124-137.

Tennessen, J.A., Bigham, A.W., O'Connor, T.D., Fu, W., Kenny, E.E., Gravel, S., McGee, S., Do, R., Liu, X., Jun, G., Kang, H.M., Jordan, D., Leal, S.M., Gabriel, S., Rieder, M.J., Abecasis, G., Altshuler, D., Nickerson, D.A., Boerwinkle, E., Sunyaev, S., Bustamante, C.D., Bamshad, M.J., Akey, J.M., 2012. Evolution and functional impact of rare coding variation from deep sequencing of human exomes. Science $337,64-69$.

Wallace, B.C., Schmid, C.H., Lau, J., Trikalinos, T.A., 2009. Meta-Analyst: software for meta-analysis of binary, continuous and diagnostic data. BMC Med. Res. Methodol. 9, 80 . 


\section{Figure legends}

Figure 1:

Forest plot with the rs7593268-T allelic odds ratios from published studies and overall odds ratio.

Suppl. Figure 1:

Forest plot showing the effect of the $A P O E$ - $\varepsilon 4$ allele on the association of $\mathrm{p} . \mathrm{R} 47 \mathrm{H}$ variant with Alzheimer's disease in different populations and overall OR comparing $A P O E-\varepsilon 4$ carriers and non-carriers. 
Table 1. Frequency of TREM2 p.R47H and $A P O E-\varepsilon 4$ carriers across phenotypes.

\begin{tabular}{lll}
\hline & TREM2 p.R47H & APOE- $\varepsilon 4$ \\
& $\mathrm{~N}($ frequency $\%)$ & $\mathrm{N}($ frequency $\%)$ \\
\hline Controls & $3(0.14)$ & $257(16.1)$ \\
$\mathrm{AD}$ & $18(0.57)$ & $1,264(44.8)$ \\
FTD & - & $64(24.5)$ \\
OR $[95 \% \mathrm{CI}](\mathrm{AD}$ vs C) & $4.12[1.21-14.00], P=0.014$ & $4.23[3.61-4.93], P=6.9 \times 10^{-83}$ \\
OR [95\%CI] (FTD vs C) &,$- P=1$ & $1.69[1.24-2.31], P=0.001$ \\
\hline
\end{tabular}

